\title{
Vestibular involvement in spasmodic torticollis
}

\author{
ADOLFO M BRONSTEIN, PETER RUDGE \\ From the Medical Research Council Neuro-Otology Unit, Institute of Neurology, National Hospital for Nervous \\ Diseases, London, UK
}

SUMMARY Vestibular findings in a group of 35 patients with spasmodic torticollis without other otological or neurological symptoms were reviewed. The most consistent abnormality, present in more than $70 \%$ of cases, was a directional preponderance of vestibular nystagmus in the dark in a direction opposite to the head (chin) deviation. Rigidly clamping the head to a rotating chair did not abolish the directional preponderance. In the presence of optic fixation the directional preponderance was less frequent and its severity tended to diminish as a function of the duration of the disease. Smooth pursuit and optokinetic nystagmus were only occasionally affected. The results are indicative of primary involvement of the vestibular system in spasmodic torticollis and are discussed in terms of a break-down of the central mechanisms conveying sensory information responsible for head and eye orientation.

Spasmodic torticollis is a motor disorder comprising involuntary activation of the neck muscles resulting in intermittent or persistent deviation of the head. Although the underlying pathophysiology is unknown, the disorder is usually considered to be one of the extra-pyramidal system particularly involving the basal ganglia. On the other hand, in animals vestibular lesions cause striking postural derangement of the head position ${ }^{1}$ and in man there are sporadic reports of vestibular abnormalities in patients with spasmodic torticollis. ${ }^{2-6}$ Indeed operations on the vestibular system have been used as a treatment for spasmodic torticollis in man. ${ }^{46}$ It is not clear, however, whether the vestibular abnormalities found in patients with spasmodic torticollis are primarily causative or the result of the abnormal head posture or the result of abnormal interaction with other sensorymotor systems.

In an attempt to clarify this we have looked at two groups of patients with spasmodic torticollis in whom extensive vestibular investigations were undertaken. The first included all the patients with spasmodic torticollis referred to the MRC Neuro-Otology Unit at the National Hospital, whose results will be discussed here. The second group comprises three patients each of whom developed spasmodic torticollis in associ-

Address for reprint requests: Dr P Rudge, The National Hospital for Nervous Diseases, Queen Sq, London WCIN 3BG, UK.

Received 26 April 1985 and in revised form 2 July 1985. Accepted 6 July 1985 ation with an 8 th nerve disturbance and will be considered in a separate paper.

\section{Material and methods}

Clinical records of 71 patients with torticollis seen in the MRC Neuro-Otology Unit at the National Hospital between 1957 and 1984 were reviewed. As it was intended thato abnormalities found on vestibular testing should be strictly related to spasmodic torticollis, all cases with additionaê neurological signs were excluded (28 cases). Most of these 28 patients had focal or generalised dystonic features, writer's cramp, Parkinsonism, tremor, pyramidal lesions or cerebrovascular disease. Five patients with otological diseases were also excluded as were six additional patients. Of these latter six, two had pure retrocollis, two had psychogenic torticollis, one had incomplete medical records and one had tuberculosis of the cervical vertebrae. Three patients had more than one condition resulting in their exclusion.

The clinical material thus comprises 35 patients ( 18 female and 17 male). The mean age at the time of the neurootological examination was 46 years (24 to 70 years) with a mean duration of spasmodic torticollis of 4 years ( 10 weeks to 19 years). The head could be rotated or tilted in a tonic or spasmodic manner; the most frequent abnormality was a combination of these four conditions. The direction of the torticollis was specified by the chin position relative to the mid line, that is chin to the right is right torticollis.

Clinical neuro-otological evaluation included assessment of the Romberg test and gait (eyes open and closed). Any nystagmus in primary position or with gaze deviation to right, left, up and down was noted. The Hallpike manoeuvre was used to produce positional nystagmus. Optokinetic nystagmus (OKN) was elicited with a small black and white striped drum rotated in the vertical and horizontal planes. 
Smooth pursuit eye movements were assessed clinically in 15 patients. Caloric tests were performed in the presence of visual fixation with the head held in the primary position in 31 patients. Methods and criteria used to establish abnormalities of the various caloric patterns were as in Fitzgerald and Hallpike; ${ }^{7}$ nine patients had, in addition, caloric testing in the dark while their eye movements were observed either with an infra-red viewer or with Frenzel's glasses. Four patients did not undergo caloric testing.

In 24 patients electro-nystagmography (ENG) had been obtained with bitemporal electrodes and direct current amplification. The paper recordings were available and reevaluated. All the recordings included traces on primary gaze and on $30^{\circ}$ deviation to the right and the left both in the presence of visual fixation and in darkness. OKN was investigated either with a small drum or full field stimulation in 18 patients. Smooth pursuit was assessed in 13 cases. OKN and smooth pursuit were inspected visually from the paper recordings and, when in doubt, slow phase velocity was measured by hand. Labyrinthine responses to an impulsive rotation to a velocity of $40^{\circ} / \mathrm{s}$ were assessed in 13 patients and the duration of the nystagmic response to the start and the stop impulse was measured. During ENG recording attempts were made to restrict head movement as much as possible by providing the subjects with a chin and a head rest. However, as this was not always entirely satisfactory, five patients were additionally tested on another revolving chair in which, by means of a head rest and bi-auricular head clamp, complete head immobility was achieved. These patients underwent sinusoidal rotation in the dark at a frequency of $0.3 \mathrm{~Hz}$ and peak velocity of approximately $50 \%$ s.

\section{Results}

\section{(a) Clinical assessment}

Body balance was largely preserved in spasmodic tor- ticollis; Romberg's test was negative in all 35 patients and gait was normal in 31 . There was minimal lateropulsion in four patients $(11 \%)$ on walking with the eyes closed. This body deviation was in the same direction of torticollis in one case and opposite in the other 3 .

A summary of the vestibulo-oculomotor findings and their relation to the side of the neck torsion is presented in table 1 . Spontaneous nystagmus on primary gaze $\left(2 \mathrm{nd}^{\circ}\right)$ was never found and $1 \mathrm{st}^{\circ}$ nystagmus was exceptional. When present, however, it was of vestibular type and was usually apparent when the patients looked in the opposite direction to their torticollis. Positional nystagmus was seen in only two cases; it was in the direction opposite the torticollis. Of some interest were the five cases whose torticollis was markedly influenced by positional changes being more prominent in the upright position in two patients and while lying down in three. OKN was essentially normal although in $23 \%$ of cases a slight directional preponderance in the direction opposite the torticollis was found. Smooth pursuit was normal in the 15 patients examined.

\section{(b) Electronystagmography}

The results are summarised in Table 2. In 11 out of 24 cases $(46 \%)$ a vestibular nystagmus in the direction opposite to that of the torticollis was clearly seen in the dark. In eight out of these 11 cases it was found on both eccentric and primary gaze $\left(2 \mathrm{nd}^{\circ}\right)$; in the other three the nystagmus was of $1 \mathrm{st}^{\circ}$ only. The nystagmus was usually abolished on fixation. Less frequently ipsilateral or bilateral nystagmus was found. Although

Table 1 Abnormalities in clinical vestibular testing

\begin{tabular}{|c|c|c|c|c|c|c|c|c|}
\hline & \multirow{2}{*}{$\begin{array}{l}\text { Spontaneous } \\
\text { nystagmus } \\
n=35\end{array}$} & \multirow{2}{*}{$\begin{array}{l}\text { Positional } \\
\text { nystagmus } \\
n=35\end{array}$} & \multirow[t]{2}{*}{$\begin{array}{l}O K N \underset{n}{n=35} \\
n=1\end{array}$} & \multirow[t]{2}{*}{$\begin{array}{l}S P \\
n=15\end{array}$} & \multicolumn{2}{|c|}{$\begin{array}{l}\text { Caloric test (Fixation) } \\
n=31\end{array}$} & \multicolumn{2}{|c|}{$\begin{array}{l}\text { Caloric test (Darkness) } \\
n=9\end{array}$} \\
\hline & & & & & $\overline{D P}$ & $C P$ & $\overline{D P}$ & $C P$ \\
\hline $\begin{array}{l}\text { Ipsilateral } \\
\text { Contralateral } \\
\text { Bilateral } \\
\text { Normal }\end{array}$ & $\begin{array}{r}3 \% \\
11 \% \\
0 \% \\
86 \%\end{array}$ & $\begin{array}{r}0 \% \\
6 \% \\
0 \% \\
94 \%\end{array}$ & $\begin{array}{r}6 \% \\
23 \% \\
0 \% \\
68 \%\end{array}$ & $\begin{array}{r}0 \% \\
0 \% \\
0 \% \\
100 \%\end{array}$ & $\begin{array}{r}3 \% \\
39 \% \\
0 \% \\
26 \%\end{array}$ & $\begin{array}{r}13 \% \\
19 \% \\
0 \% \\
26 \%\end{array}$ & $\begin{array}{r}0 \% \\
78 \% \\
0 \% \\
11 \%\end{array}$ & $\begin{array}{r}11 \% \\
0 \% \\
0 \% \\
11 \%\end{array}$ \\
\hline
\end{tabular}

$\mathbf{n}=$ number of patients examined.

Ipsilateral-contralateral $=$ abnormality in the same-opposite direction of torticollis in this and subsequent tables.

OKN = optokinetic nystagmus.

$\mathrm{DP}=$ directional preponderance of nystagmus.

$\mathrm{CP}=$ canal paresis.

SP = smooth pursuit.

Table 2 ENG abnormalities

\begin{tabular}{|c|c|c|c|c|c|}
\hline & \multicolumn{2}{|c|}{ Spontaneous nystagmus $n=24$} & \multirow{2}{*}{$\begin{array}{l}O K N D P \\
n=18\end{array}$} & \multirow{2}{*}{$\begin{array}{l}S P \\
n=13\end{array}$} & \multirow{2}{*}{$\begin{array}{l}\text { Rotation } \\
n=13\end{array}$} \\
\hline & $\overline{\text { Light }}$ & Dark & & & \\
\hline $\begin{array}{l}\text { Ipsilateral } \\
\text { Contralateral } \\
\text { Bilateral } \\
\text { Normal }\end{array}$ & $\begin{array}{r}0 \% \\
0 \% \\
4 \% \\
96 \%\end{array}$ & $\begin{array}{l}12 \% \\
46 \% \\
17 \% \\
25 \%\end{array}$ & $\begin{array}{r}5 \% \\
39 \% \\
0 \% \\
55 \%\end{array}$ & $\begin{array}{r}0 \% \\
15 \% \\
0 \% \\
85 \%\end{array}$ & $\begin{array}{r}23 \% \\
76 \% \\
0 \% \\
0 \%\end{array}$ \\
\hline
\end{tabular}


in more than half of the cases OKN was normal, a directional preponderance opposite to the torticollis was encountered in $39 \%$. Smooth pursuit was normal in most patients tested; in two out of $13(15 \%)$ patients pursuit was moderately broken (saccadic) in the direction opposite to the torticollis.

\section{(c) Rotational and caloric testing}

Labyrinthine function assessed by the caloric test or rotation was usually abnormal. Only eight of 31 patients $(26 \%)$ in whom caloric test was performed in the presence of fixation and one of the nine patients $(11 \%)$ who were also assessed in the dark had normal responses to irrigation. There was a caloric directional preponderance in the direction opposite to the torticollis in $33 \%$ of the patients in the light and $78 \%$ of patients in the dark. Other abnormalities, listed in table 1 , were less frequent.

None of the 13 patients in whom rotational tests were done had a normal symmetrical response (table 2). $76 \%$ of the patients had a clear directional preponderance in the direction opposite the torticollis, a figure in good agreement with that obtained with caloric testing in the dark (78\%).

An attempt was made to correlate the degree of asymmetry found in the vestibular tests with features of the disease. Unfortunately we had no reliable way of assessing the severity of spasmodic torticollis. However the duration of the disorder seemed to be inversely related to the degree of asymmetry found on caloric testing. Since the duration of caloric and rotational nystagmus was available in all cases a directional preponderance index was calculated using the following formula:

Duration of induced nystagmus in the direction of torticollis $\left(d_{s}\right)$

Duration of induced nystagmus in the direction opposite to the torticollis $\left(d_{o}\right)$

From this it can be seen that if, $d_{s} / d_{o}<1$ the directional preponderance was opposite to torticollis while if $d_{s} / d_{o}>1$ the directional preponderance is in the same direction of torticollis; $d_{s} / d_{o}=1$ indicates symmetric responses.

The directional preponderance indices of 29 out of 31 cases who had caloric testing in the light were calculated; two cases had to be discarded because in one of them the caloric test had been repeated with contradictory results and, in the other, only one of the four irrigations induced nystagmus. The directional preponderance indices of caloric test in the light of spasmodic torticollis patients with less than a year's duration were $0 \cdot 77, S D=0 \cdot 17(n=10)$, between 1 to 5 years $=0.92, \mathrm{SD}=0.13(\mathrm{n}=11)$ and more than 5 years $=0 \cdot 97, S D=0 \cdot 11(n=8)(f i g 1)$. There was a

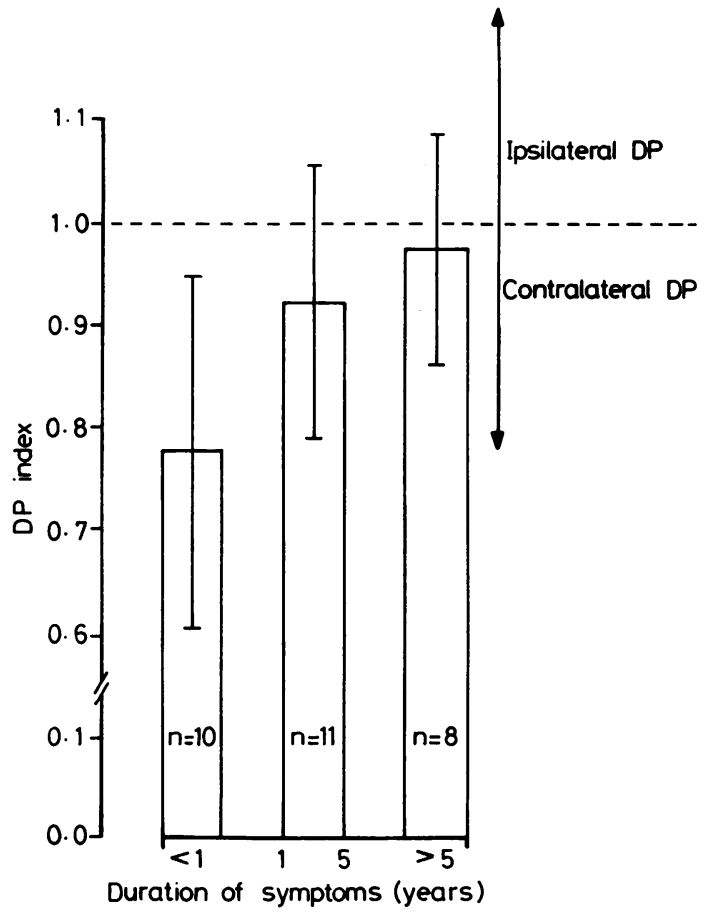

Fig 1 Relationship between duration of torticollis and directional preponderance $(D P)$ indices of caloric test in the light. Means and $S D$ are shown.

positive logarithmic correlation between the duration of the illness and directional preponderance index (e $=0.55 ; \mathrm{p}<0.01)$. This indicates that spasmodic tor $=$ ticollis patients show a tendency to have more active nystagmus (directional preponderance) in a direction opposite to the torticollis and that this asymmetry, in the presence of optic fixation, tends to decrease with time.

Further analysis of these data was undertaken. If only those patients who had rotational or caloric directional preponderance in the direction opposite to torticollis are considered (17 of the original 31) a better inverse correlation between the duration of the torticollis and the directional preponderance is found $r=0.75 p<0.01$ (fig 2). On the other hand, in the same group there was no correlation between the directional preponderance obtained on caloric testing in the dark or on rotational testing and the duration of the illness (fig 3). These data indicate that although directional preponderance in the presence of fixation shows a progressive reduction with time, the directional preponderance in the dark remains unchanged.

In order to establish whether the directional preponderance was a trivial consequence of the head movement or posture five patients (plus one with ad- 


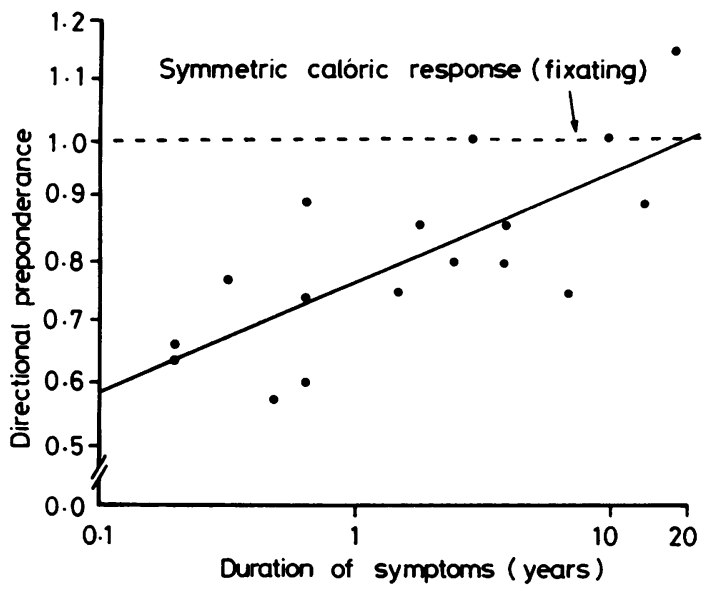

Fig 2 Relationship between duration of torticollis and directional preponderance indices of caloric test in the light. Only the patients who had a rotational or caloric directional preponderance contralateral to the torticollis are presented.

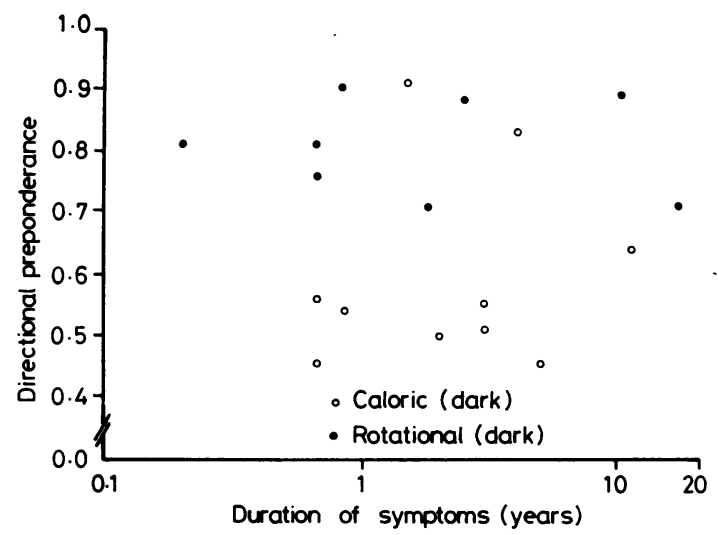

Fig 3 Relationship between duration of torticollis and directional preponderance indices in the dark in patients with caloric or rotational directional preponderance contralateral to the torticollis.

ditional mild unilateral upper limb dystonia who was not included in the present series) were subjected to sinusoidal rotation in darkness with the head completely immobilised in the central position. The gain of the vestibulo-ocular reflex (slow phase eye velocity/chair velocity) was measured separately to right and left. From fig 4, a case with left torticollis, it can be seen that nystagmus to the right was more active and of longer duration than in the opposite direction. All the other cases behaved similarly, VOR gain being higher during the half cycle in which the chair moved in a direction opposite to that of the torticollis.

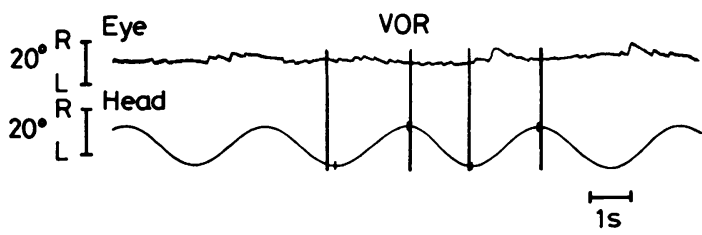

Fig 4 Eye movements in the dark during sinusoidal rotation with the head clamped (vestibulo-ocular reflex, VOR) in a patient with left torticollis. The short and long vertical lines indicate respectively the points at which the head and the slow component of the eye movement reverse direction. During rotation to the left the eyes are advanced relative to the head (phase lead indicated by the larger gap between short and long vertical lines). Thus, hemicycles of right beating nystagmus are of greater magnitude and duration than those to the left.

\section{Discussion}

Our results show that there are neuro-otological abnormalities in patients with spasmodic torticollis. The most consistent finding is the directional preponderance of vestibular induced nystagmus opposite to the direction of the torticollis. Since the directional preponderance is specified by the fast phase of the nystagmus the vestibular induced slow phase is greater in the opposite direction to the directional preponderance and thus in the same direction as the torticollis. The same trend was noted, although not emphasised, by Matthews et al. ${ }^{8}$ Such a finding is consistent with the hypothesis that there is tonic imbalance of muscle activity both in the neck and the extra-ocular system which tends to deviate the head and the eyes in the same direction. That this directional bias of the vestibularly elicited eye movements is not a trivial consequence of the head movement is supported by three observations.

Firstly, in normal subjects, forced rotation of the head does not result in a directional preponderance of the rotational response (unpublished observations). Secondly, if the head is fixed during vestibular stimulation in a patient with torticollis the directional preponderance persists. Thirdly, if the directional preponderance were a consquence of the position of the head it might be expected to remain constant or even increase with time. In fact the reverse occurs in spite of the torticollis persisting. For these reasons we think the directional preponderance of induced nystagmus is more fundamentally related to the torticollis.

From the available evidence the type of neurootological abnormalities found indicate that the vestibular system itself, rather than other ocular-motor sub-systems, is affected. This is suggested by the much higher incidence of directional preponderance or 
spontaneous nystagmus in the dark than in the light, indicative of a preserved capacity to compensate for vestibular asymmetry by fixation, and by the relative integrity of the more purely visuo-motor tests such as smooth pursuit and to a lesser degree OKN.

It might be argued, based on some isolated reports in the clinical literature, that the torticollis and directional preponderance stem from a vestibular end organ, 8th nerve or vestibular nuclear disturbance. Although this is a superficially attractive hypothesis, it is unlikely to be true since the vast majority of cases of spasmodic torticollis have no overt 8th nerve symptomatology.

It is more likely that the neuro-otological abnormalities are due to a central disturbance of posture which also affects the tonus balance of the vestibuloocular system. In Parkinson's disease, for instance, abnormalities of caloric, ${ }^{9}$ rotational ${ }^{10}$ and tilting reactions ${ }^{11}$ have been reported which could be explained on a similar basis. Unfortunately, however, unilateral cases were not presented separately in order to determine whether basal ganglia diseases other than torticollis are able to produce a directional preponderance in the vestibular tests.

The vestibular system has complex connections within the brain stem, thalamus and cortex where interaction with other sensory modalities can occur. $^{12-17}$ It is possible that the abnormality in spasmodic torticollis is a break down of these central connections and particularly their interaction with other modalities signalling posture of the somatic musculature controlling head and eye position. Such a break down is consistent with a perverted or exaggerated response to sensory stimuli in patients with spasmodic torticollis and would account for the observations that patients are able to control their head position by cutaneous stimulation, have increased sensitivity to proprioceptive input ${ }^{18}$ and for the effect of position with respect to gravity seen in five of our cases. The possibility that in some cases an abnormal peripheral labyrinthine input might play a role in the genesis of this break down is, however, an open question currently being investigated.

It is apparent from the present series that the directional preponderance in the direction opposite to the chin deviation, although frequently found, is not a constant feature of the disease. We suggest two possible reasons for this. Firstly, there is evidence from stereotaxic surgery that different forms of spasmodic torticollis (jerky, tonic, rotational, tilted) have a distinctive anatomical basis. ${ }^{19}$ It is thus possible that different vestibular pathways are involved in various types of spasmodic torticollis. Secondly, the type and degree of compensation of the vestibular derangement could vary. Although this is entirely speculative there is no doubt that such compensation mech- anisms are active in patients with spasmodic torticollis at least as far as the vestibulo-ocular system is concerned, in that removal of fixation in our study, resulted in an asymmetry of the vestibular ocular reflex in many patients. The exact nature of these complex derangements and corrective processes, however, remains as obscure as when Barre ${ }^{2}$ proposed a similar mechanism over sixty years ago.

Dr Bronstein is supported by a grant from the Brain Research Trust.

Our gratitude goes to Dr MR Dix and Dr JD Hood for useful suggestions and criticisms during the preparation of this work.

\section{References}

${ }^{1}$ Magnus R, Korperstellung Berlin: Verlag Von Julius Springer, 1924.

2 Barre JA. Le Torticolis Spasmodique. Rev Neurol (Paris) (I) 1929:984-1013.

${ }^{3}$ Barre JA, Guillaume J. Torticolis spasmodique; troubles vestibulaires unilateraux. Remarques cliniques et chirurgicales. Rev Neurol (Paris) (I) 1930:485-7.

${ }^{4}$ Hyndman OR. Torticollis spastica. Arch Otolaryngoto 1939;29:827-38.

${ }^{5}$ Snyder $\mathrm{CH}$. Paroxysmal torticollis in infancy. A possible form of labyrinthitis. Am J Dis Child 1969;117:458-505

${ }^{6}$ Svien HJ, Cody DTR. Treatment of spasmodic torticollis by suppression of labyrinthine activity: report of a case Mayo Clin Proc 1969;44:825-7.

${ }^{7}$ Fitzgerald G, Hallpike CS. Studies in human vestibular function: I Observations on the directional prepon derance of caloric nystagmus resulting from cerebraf lesions. Brain 1942;65:115-80.

${ }^{8}$ Matthews WB, Beasley P, Parry-Jones W, Garland G. Spasmodic torticollis: a combined clinical study. J Neurology Neurosurg Psychiatry 1978;41:485-92.

${ }^{9}$ Reichert WH, Doolittle J, McDowell FH. Vestibular dysfunction in Parkinson disease. Neurology $(N Y)$ 1982;32:1113-8.

${ }^{10}$ White OB, Saint-Cyr JA, Sharpe JA. Ocular motor deficits in Parkinson's disease. I. The horizontal vestibulo-ocular reflex and its regulation. Brain 1983;106:555-70.

${ }^{11}$ Martin JP. Tilting reactions and disorders of the Basal Ganglia. Brain 1965;88:855-74.

${ }^{12}$ Muskens LJJ. The central connections of the vestibular nuclei with the corpus striatum and their significance for ocular movements and for locomotion. Brain 1922;45:454-78.

${ }^{13}$ Montandon P. Functional correlations between the diencephalic nystagmogenic area and the vestibular nystagmogenic area. Acta Otolaryng (Stockh) 1964;Suppl 186.

${ }^{14}$ Denny-Brown D. Clinical symptomatology of diseases of the basal ganglia (chap 5). In: Vinken PJ, Bruyn GW, eds. Handbook of Clinical Neurology, vol 6. Amsterdam: North Holland, 1968:133-73. 
${ }^{15}$ Spiegel ED, Szekely EG, Gildenberg PL. Vestibular responses in midbrain, thalamus and basal ganglia. Arch Neurol 1965;12:258-69.

${ }^{16}$ Potegal M, Copack P, de Jong JMBV, Krauthamer G, Gilman S. Vestibular input to the caudate nucleus. Exp Neurol 1971;32:448-65.

${ }^{17}$ Shima F. Circling behavior depending on striopallidal and vestibular functions. In: Hassler RG, Christ JF, eds. Advances in Neurology. New York: Raven Press, 1984;40:47-54.

${ }^{18}$ Podivinsky F. Torticollis (chap 23). In: Vinken PJ, Bruyn GW, eds. Handbook of Clinical Neurology, vol 6. Amsterdam: North Holland, 1968:567-603.

${ }^{19}$ Hassler R, Dieckmann G. Stereotactic treatment of different kinds of Spasmodic Torticollis. Confin Neurol 1970;32:135-43. 